\title{
Desert Research and Technology Studies 2008 Report
}

\author{
Barbara Romig, Joseph Kosmo, Michael Gernhardt \\ National Aeronautics and Space Administration, Johnson Space Center
}

Andrew Abercromby

Wyle Laboratories

Copyright (c) 2008 SAE International

\begin{abstract}
During the last two weeks of October 2008, the National Aeronautics and Space Administration (NASA) Johnson Space Center (JSC) Advanced Extravehicular Activity (AEVA) team led the field test portion of the 2008 Desert Research and Technology Studies (D-RATS) near Flagstaff, AZ. The Desert RATS field test activity is the year-long culmination of various individual science and advanced engineering discipline areas' technology and operations development efforts into a coordinated field test demonstration under representative (analog) planetary surface terrain conditions.
\end{abstract}

The 2008 Desert RATS was the eleventh RATS field test and was the most focused and successful test to date with participants from six NASA field centers, three research organizations, one university, and one other government agency. The main test objective was to collect Unpressurized Rover (UPR) and Lunar Electric Rover (LER) engineering performance and human factors metrics while under extended periods of representative mission-based scenario test operations involving long drive distances, night-time driving, Extravehicular Activity (EVA) operations, and overnight campover periods.

The test was extremely successful with all teams meeting the primary test objective. This paper summarizes Desert RATS 2008 test hardware, detailed test objectives, test operations, and test results.

\section{INTRODUCTION}

Desert RATS is a combined group of inter-NASA center engineers and scientists, collaborating with representatives of industry and academia, for the purpose of conducting remote field exercises. These remote field exercises provide the capability to 1 ) identify and establish technical requirements applicable for future planetary exploration, 2) validate mission operational techniques, and 3) test experimental hardware and software. [1]

The primary objective of Desert RATS is to test preliminary exploration operations concepts to drive out EVA system requirements by providing hands-on experience with simulated planetary surface exploration EVA hardware and procedures. RATS activities also are of significant importance in helping to develop the necessary levels of technical skills and experience for the next generation of engineers, scientists, technicians, and astronauts who will be responsible for realizing the goals of the Constellation Program. In order to learn how to be a good engineer, there is no substitute for the hands-on experience that RATS provides. [1]

\section{PARTICIPANTS}

Participation in the remote field test has grown and fluctuated in size over its 11 year span. It started in 1997 with 4 people, has been over 100 people in 2006 and 2007 , and was over 50 people in 2008 . These numbers do not include countless other team members who support the hardware and test development throughout the year who do not attend the remote field test. The participants provide expertise, hardware, and momentum for Desert RATS, and they have been recruited or have joined the effort based on their skills and expertise that are required to meet the test objectives.

2008 participants were from six NASA field centers (JSC, Kennedy Space Center (KSC), Ames Research Center (ARC), Langley Research Center (LaRC), Glenn Research Center (GRC), and Headquarters (HQ)), three research organizations (Smithsonian Institution, Mars Institute, and Lunar Planetary Institute (LPI)), one university (Arizona State University (ASU)), and one other government agency (United States Geologic Survey (USGS)). Table 1 lists the participants and their contributions to Desert RATS in 2008. Figure 1 shows a portion of the personnel that participated in the field in 2008. 


\begin{tabular}{|c|c|}
\hline Participant & Contribution(s) \\
\hline \multicolumn{2}{|l|}{ NASA Centers: } \\
\hline JSC & $\begin{array}{l}\text { - Led/Coordinated Desert RATS } 2008 \\
\text { - Test hardware: Chariot chassis configured as UPR, Chariot chassis with cabin } \\
\text { - } \text { Configured as LER, suit ports, mockup suits, EVA geology tools } \\
\text { - } \quad \text { Human factors and other data collection } \\
\text { - } \text { Lead for science team and traverses } \\
\text { - } \quad \text { Public Affairs Office (PAO) } \\
\text { - } \text { Medical and test safety support for field team } \\
\text { - Photography and videography support }\end{array}$ \\
\hline KSC & $\begin{array}{l}\text { - Test site communications and networking, site aggregate backhaul } \\
\text { - Team lead for camp and power layout, logistics support } \\
\text { - Suit camera lead }\end{array}$ \\
\hline ARC & $\begin{array}{l}\text { - Science team support (in collaboration with the Mars Institute) } \\
\text { - } \quad \text { Supported initial concept development for LER suit ports }\end{array}$ \\
\hline LaRC & $\begin{array}{ll}\text { - } & \text { NASA EDGE coverage } \\
\text { - } \quad \text { LER cabin composite closeout panels \& honeycomb aft bulkhead structure }\end{array}$ \\
\hline GRC & - Exercise ergometer for LER \\
\hline $\mathrm{HQ}$ & $\begin{array}{l}\text { - PAO lead } \\
\text { - Overall project management }\end{array}$ \\
\hline \multicolumn{2}{|l|}{ Research Organizations: } \\
\hline Smithsonian Institution & $\begin{array}{l}\text { - Science team support } \\
\text { - } \quad \text { Geologist suit subject (crew A) }\end{array}$ \\
\hline Mars Institute & $\begin{array}{l}\text { - Science team support (in collaboration with ARC) } \\
\text { - Geologist suit subject (crew B) }\end{array}$ \\
\hline LPI & - Science team support \\
\hline \multicolumn{2}{|l|}{ University: } \\
\hline ASU & - $\quad$ Science team support \\
\hline \multicolumn{2}{|l|}{ Government Agency: } \\
\hline USGS & $\begin{array}{ll}\text { - } & \text { Test site identification, characterization, and permits } \\
\text { - } & \text { Logistics base and support }\end{array}$ \\
\hline
\end{tabular}

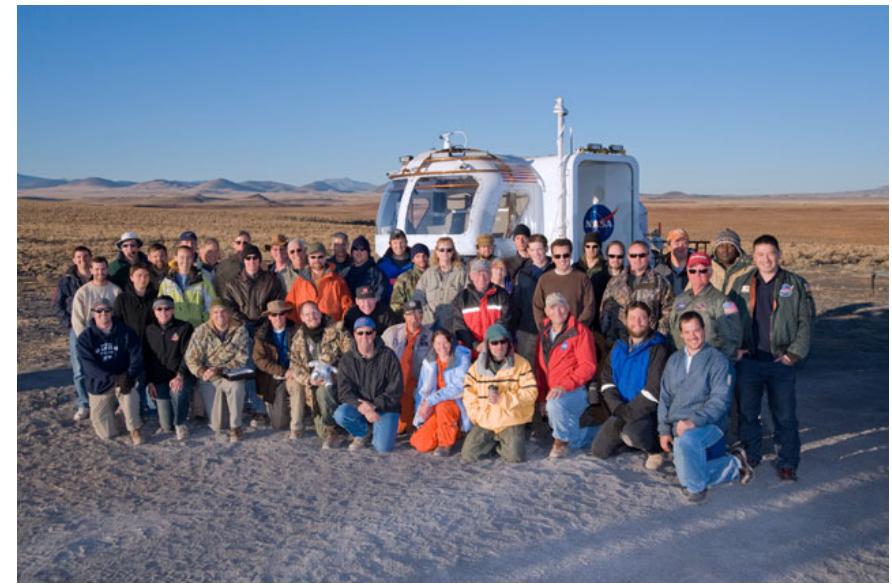

Figure 1 (jsc2008e138698): 2008 Test Team

\section{TEST LOCATION}

The Rock Yard located at JSC is ideal for conducting routine "dry runs" and checkouts of individual systems and interactive test activities on a small scale. It consists of three main areas: the rock yard, Mars hill, and the lunar yard. Figure 2 is an aerial view of the JSC Rock Yard.

The rock yard is composed of stabilized crushed limestone and decomposed granite base covered with strewn basaltic rocks. It is approximately $42.7 \mathrm{~m} \times 42.7$ $\mathrm{m}(140 \mathrm{ft} \times 140 \mathrm{ft})$. Mars hill is composed of stabilized crushed limestone and decomposed granite base covered with strewn basaltic rocks. Its footprint is approximately $42.7 \mathrm{~m} \times 42.7 \mathrm{~m}(140 \mathrm{ft} \times 140 \mathrm{ft})$. The lunar yard is composed of stabilized crushed limestone base covered with blackstar granite. It is approximately $42.7 \mathrm{~m} \times 42.7 \mathrm{~m}(140 \mathrm{ft} \times 140 \mathrm{ft})$ and contains three representative Lunar craters [2]. There is also an extended area consisting of calibrated sand and excavated dirt for challenging the robotic elements and for practicing site preparation (bulldozing, etc.). 


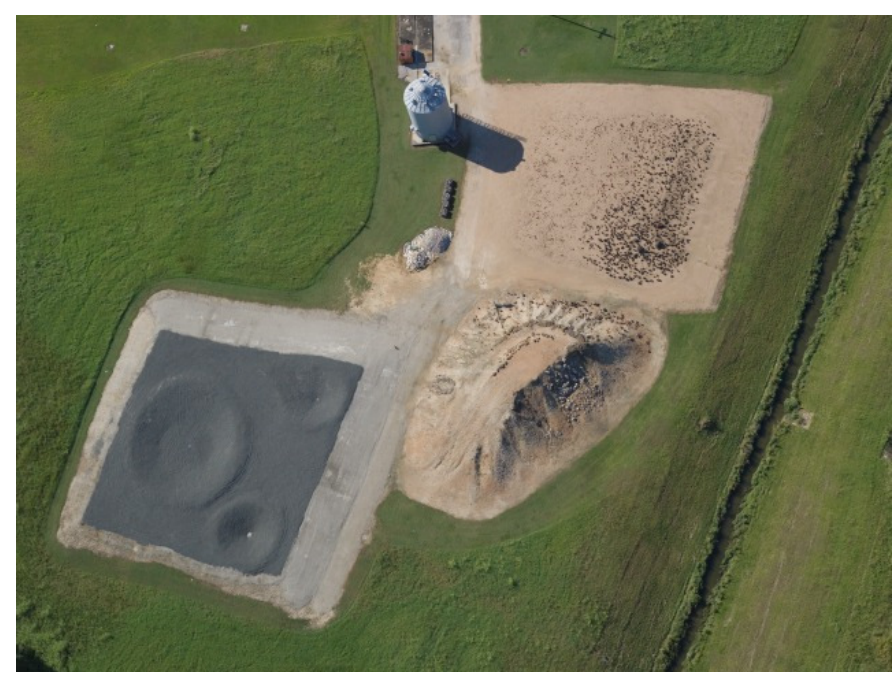

Figure 2 (jsc2007e052145): Aerial photo of JSC Rock Yard

Geologically relevant terrain is necessary to assess the ability of crewmembers to make geological contextual observations from inside the LER compared with the UPR. It is also required to enable estimation of LER performance, safety, and productivity metrics, such as number of EVAs, maximum separation of EVA astronaut and LER, boots-on-surface EVA time, and average drive speed, all of which are largely terrain dependent.

The Black Point Lava Flow (BPLF) test site offers a large scale test area ( 750,000 acres) approximately $65 \mathrm{~km}$ $(\sim 40 \mathrm{mi})$ from Flagstaff, AZ. The BPLF has a wide variety of surface features with respect to geological relevance. The geological features (numerous outcrop and contact lines) and terrain (particularly along the edge of the lava flow) provide many opportunities to evaluate the Intravehicular Activity (IVA) and EVA science/exploration capabilities of the LER in single-day or multi-day science-driven sorties. The size of the BPLF site and the abundance of geological features would enable extended range science-driven LER sorties (potentially $>100 \mathrm{~km}+$ driven distance with 7-14 days of operational time). Figure 3 is an aerial view of the BPLF.

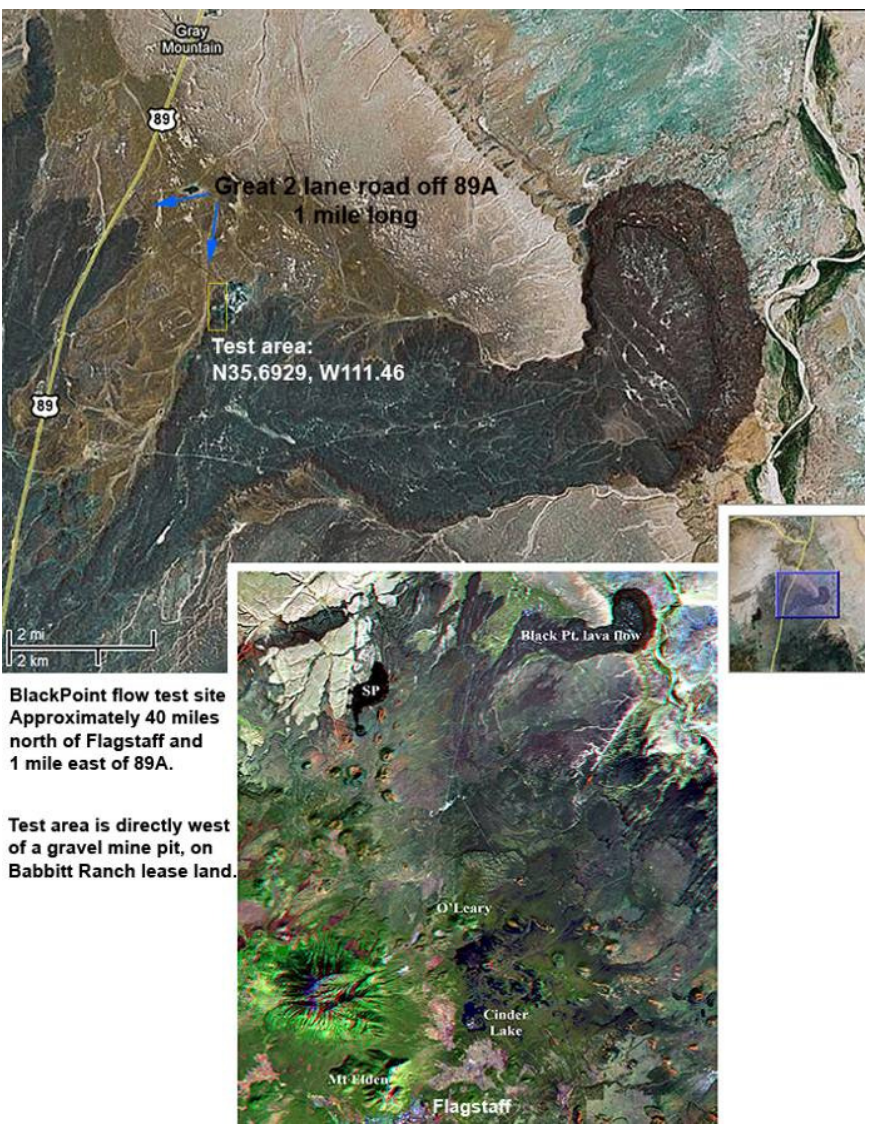

Figure 3: Aerial view of Black Point Lava Flow, $A Z$

\section{TEST HARDWARE DESCRIPTIONS}

\section{CHARIOT MOBILITY CHASSIS}

NASA JSC's Automation, Robotics, and Simulation Division (ER) in the Engineering Directorate (EA) provided the Chariot chassis, or lunar truck.

Chariot has active suspension, six-wheel drive with independent steering for each wheel, and crab steering that allows each wheel to pivot individually in any direction, regardless of where any other wheel points. Chariot can also raise or lower individual wheels to keep the vehicle level when driving on uneven ground or to lower the belly to the ground. Chariot is a concept vehicle that was designed to provide a mobile platform to develop and test advanced rover technologies and to enable the development and demonstration of mission operations and concepts applicable to future planetary rover vehicle development activities. [3] 


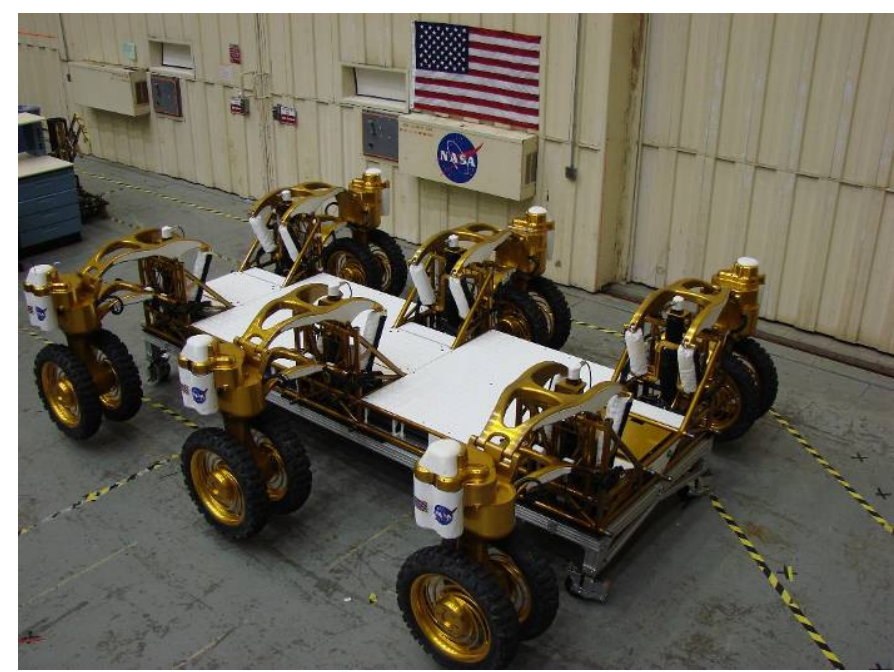

Figure 4: Chariot chassis, or Lunar Truck

\section{CHARIOT CONFIGURED AS A UPR}

The UPR configuration is the Chariot chassis with two turret interfaces to space suits. Tool pallets and other payloads can be attached to the UPR for added capability. In the UPR mode, up to two EVA suited crew members are on-board. Excursions are limited to the 8 hour maximum EVA duration. NASA JSC ER provided the turret systems. Figure 4 shows the UPR.

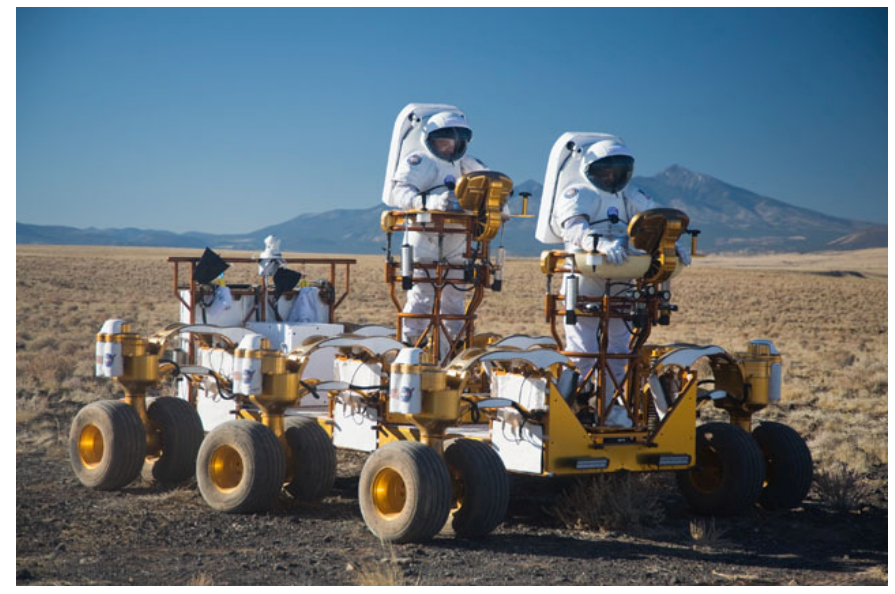

Figure 5 (jsc2008e138250): UPR Configuration

\section{CHARIOT CONFIGURED AS A LER}

The LER configuration is the addition of a cabin, including an aft bulkhead with suit ports, to the Chariot chassis. The LER cabin was developed as a collaborative effort between NASA JSC, LaRC, ARC, and GRC. The concept vehicle cabin is not pressurized, but will incorporate functional suit ports including alignment guides and clamping mechanisms, all of the necessary crew accommodations to support a 3-day traverse, and will be fully integrated with the Chariot chassis, consistent with the current Constellation Lunar Architecture Team (CXAT_Lunar) architectural assumptions (shown in Figure 6). The LER cabin also incorporates interior cameras and microphones that will be utilized by the JSC Usability, Testing, and Analysis Facility (UTAF) in the collection of human factors data throughout the study. [4]

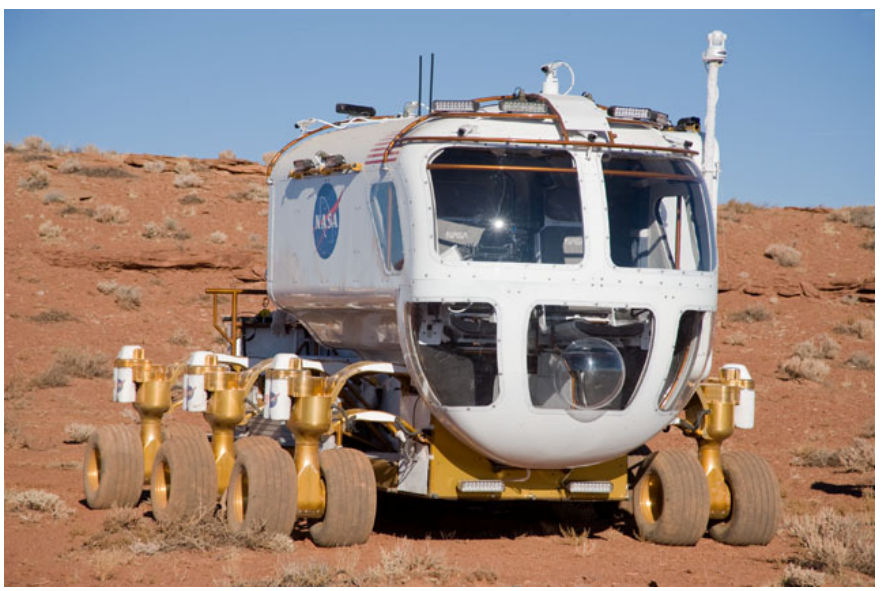

Figure 6 (jsc2008e138757): LER Configuration of cabin integrated with chassis

\section{LER SUIT PORT CONCEPT EVALUATOR}

The suit port is an element of the LER by which the crew performs EVAs. The suit port concept evaluator is located on the aft bulkhead of the LER cabin structure and includes hatches, a locking (marman) mechanism, seals, interior and exterior suit don/doff aids, and exterior platforms to accommodate different crewmember heights. [5]

The human factors evaluation of the suit port concept evaluator focused on the suit don/doff capabilities and features of the suit port architecture and the suit undocking/docking from/to suit port hatch interface. However, it is important to note that:

a) The suit port does not pressurize (a functional, pressurizable engineering unit is a different deliverable to test the sealing and locking interface of the suit to suit port)

b) The mockup suits are unpressurized and were not specifically designed for use with suit ports

c) The testing was performed in a 1-g environment

d) The mockup suits and suit ports do not incorporate the additional complexities associated with an actual Portable Life Support System (PLSS) and the data, power, gas and fluid connections between the suits and the LER

Testing in a 1-g environment using unpressurized suits that are not specifically designed for use with suit ports is likely to increase the time and difficulty associated with suit port egress/ingress tasks, while the reduced complexity of the suit port concept evaluator compared with a fully-functioning pressurized version will mean that EVA overhead and technical challenges associated with this complexity will not be identified during this study. 
Results of the suit port evaluation will be presented and interpreted within the context of these limitations. Figures 7 and 8 show the aft bulkhead and suit port exterior and interior views. [4]

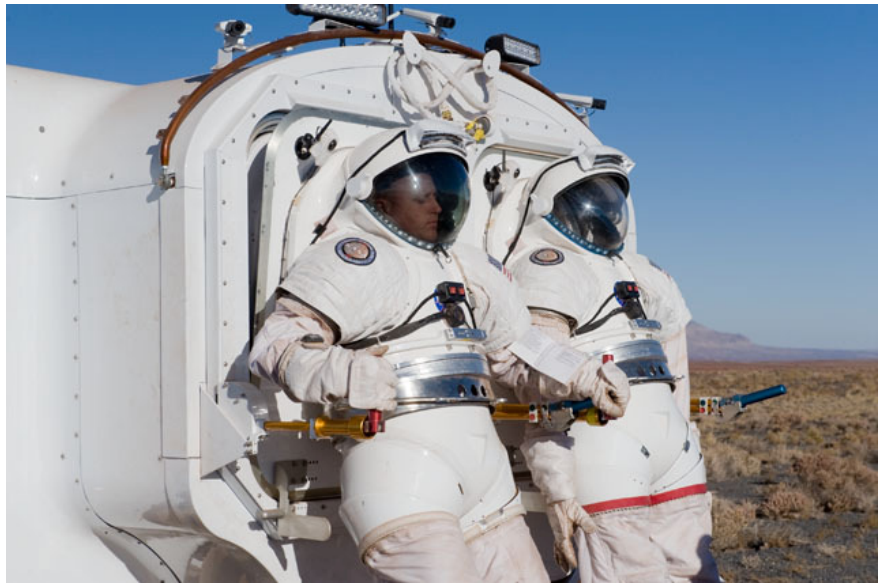

Figure 7 (jsc2008e139052): LER exterior view of aft bulkhead and suit ports with mockup suits attached

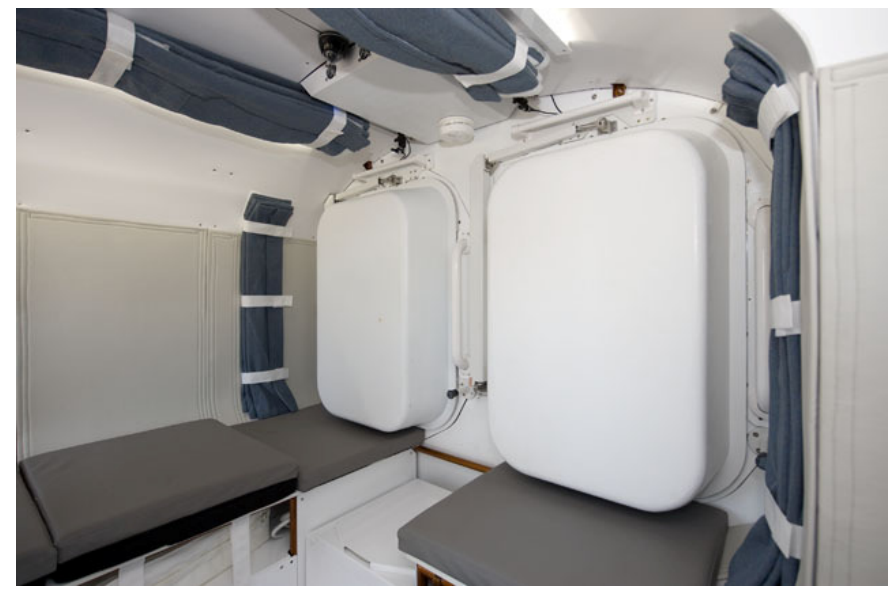

Figure 8 (jsc2008e125494): LER interior view of aft bulkhead and suit ports

\section{MARK III MOCKUP SUIT}

The Mark III suit, also called the $\mathrm{H}$-suit, represents a hybrid space suit configuration in that it is composed of hard elements such as a Hard Upper Torso (HUT) and brief, and of soft components such as single-axis fabric elbows, knees, and ankle joints. A key feature of the suit is its use of a rolling convolute waist joint and use of bearings in multi-axis mobility joint systems. The Mark III has bearings at the shoulder, upper arm, waist, upper hip, mid-hip, upper leg (3 bearing hip), and ankle joints. The suit is donned through a rear entry hatch on the HUT. Suit subjects are integrated to the suit by a waist belt weight relief system and shoulder straps. The boots of the Mark III are designed for traverses over rough terrain and were derived from a military flight-style boot. The Mark III suit weighs approximately $45.4 \mathrm{~kg}$ (100 lbs). Figure 9 shows the front of the Mark III suit as tested at the D-RATS 2007 remote field test. [6]

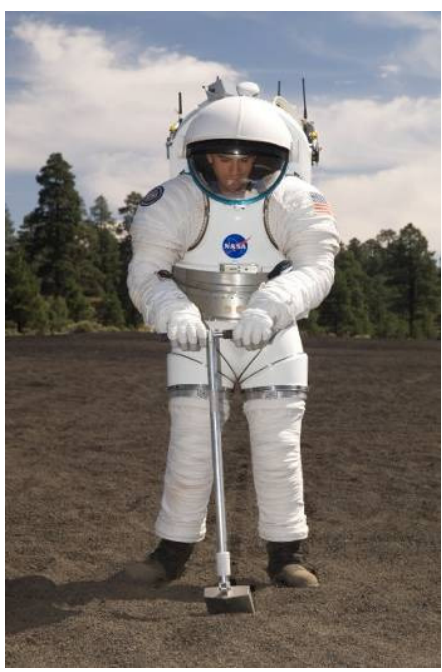

Figure 9 (jsc2007e049514): Mark III Advanced Space Suit Technology Demonstrator

This year's D-RATS testing of the comparison of a UPR and a LER required a suit that is lighter-weight than the Mark III but that looks similar (cosmetically) and that has similar mobility limitation features, such as the 3-bearing hip. Using lightweight suits minimizes injury risk and more closely represents the suit weights that will be experienced by astronauts on the lunar surface. [4]

Approximately 4 years ago, Global Effects, Inc. designed and fabricated 8 lightweight suits modeled after the Mark III to use in the movie industry. Then they fabricated a 9th suit for NASA HQ to use as a PAO display model. In April 2008, Global Effects, Inc. delivered four of the Mark III mockup suits to JSC's Crew and Thermal Systems Division (EC). [4]

The Mark III mockup suits weigh approximately $28 \mathrm{~kg}$ (62 lbs), are cosmetic representations of the Mark III suit, and have similar mobility limitations as the Mark III suit (e.g. waist bearing, hip bearings, shoulder convolutes, etc.). While the mockup suits have similar mobility limitations as the real Mark III suits, it should be noted that there are several limitations to the mockup suits as discussed in a previous section of this report. [4]

Two of the four suits were modified to interface with the LER suit port. Changes include (but are not limited to) addition of an interface plate to the rear entry hatch, increasing the PLSS volume, and adding a communication system, Global Positioning System (GPS) data logger, helmet lights, and EVA camera. Figures 10 and 11 illustrate the two Mark III mockup suit configurations. 


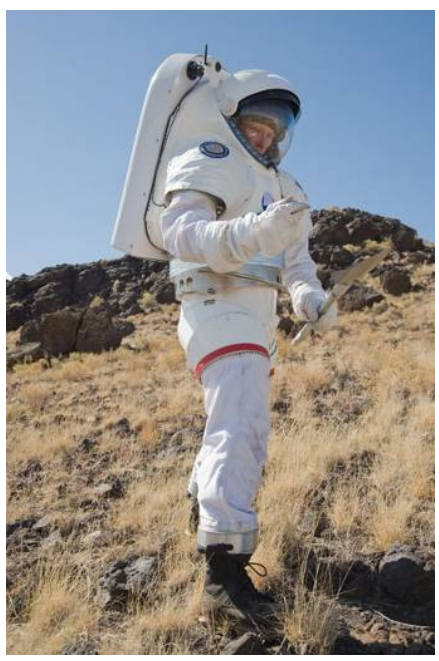

Figure 10 (jsc2008e137994): Mark III Mockup Suit, UPR Configuration

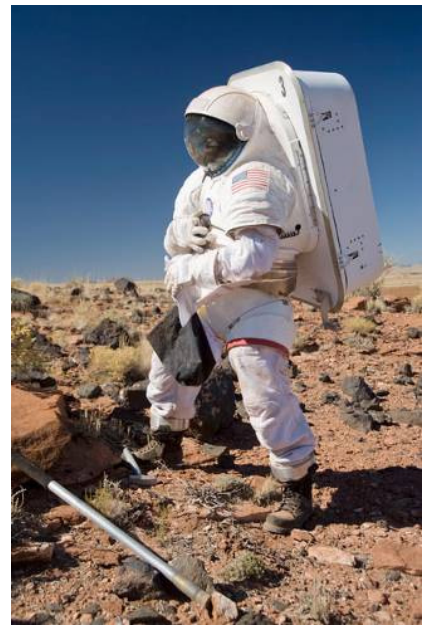

Figure 11 (jsc2008e138835): Mark III Mockup Suits, LER Configuration

\section{EVA GEOLOGY TOOLS}

To fully demonstrate the enhanced science capability of the EVA system utilizing the UPR and LER as prototype "Surface Mobility Asset" vehicles during the 2008 Desert RATS activity, a set of hand tools that facilitated field geology-style work were required. These hand tools provided geology sample gathering capability and were usable by the EVA crew. Initial capability was pursued by developing a set of Apollo-style geology tools from archive records. A simple vehicle interface was also developed, providing an EVA mounting point for storage/tool retention and easy access of the tools by the suited crew.

After examining the historic Apollo designs and implementing a few updates, a baseline set of tools was created including Large Tongs (facilitating crew members' reach), Core Tubes, a Drive Bar for manual Core Tube manipulation, Crack Hammers and Geology Hammers, an Extension Handle (adding length to Hammers and Shovels), and a Drive Head/T-Bar combination unit for the Trenching Tool and Core Tube Drive Bar. Several supporting pieces of tooling, including a Gnomen photo reference device and a walking staff, were used as well. Finally, Teflon® film Sample Bags were supplied for sample collection and curation. These bags were mounted to each EVA crewmember via a quick release "D-Ring". Filled sets of these Sample Bags as well as large samples were returned to the vehicle after collection and stowed in a softgoods containment bag that could be removed from the vehicle at the end of a sortie. This softgoods bag was mounted alongside the Science Tools at the vehicle interface known as the "Science Pallet".

The Science Pallet vehicle interface was designed to retain the tools across the vibration spectrum encountered during vehicle translation while allowing unencumbered access (both removal and re-installation) of each tool by an EVA crewmember. Simplicity and commonality in the mounting system was pursued, resulting in a reconfigurable interface that allowed 1handed removal/installation of each tool and alteration of the layout to accommodate crew preferences and future expansion of the toolkit with new science equipment. Figure 12 shows the Science Pallet with the EVA tools attached. Figure 13 shows the EVA crewmembers using some of the tools.

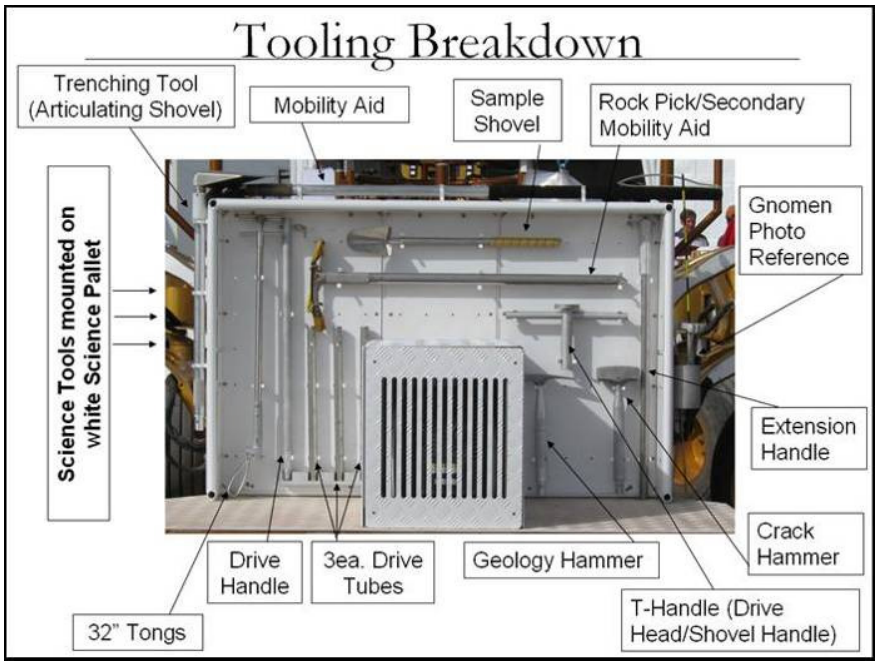

Figure 12: Science Pallet with EVA tools 


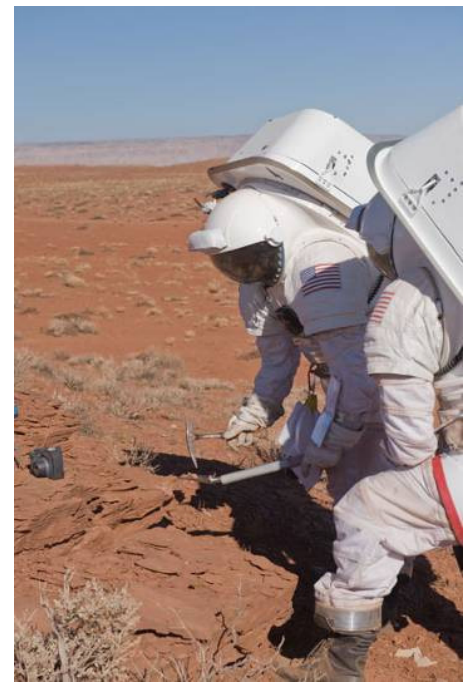

Figure 13 (jsc2008e139072): EVA crewmembers using tools

Leveraging the experience of the remaining Apollo era geologist/crew trainers and the archived engineering drawings, a new understanding and appreciation for the science community's goals and requirements was gained. Ultimately, the development of this EVA geology toolkit not only supported the planned RATS field test but enabled a deeper understanding by the contemporary EVA Tools engineering community of the historical design requirements and concept of operations for Lunar Sample acquisition with the enhanced surface mobility asset represented by the LER.

\section{LER EXERCISE ERGOMETER}

The LER incorporates a fully functional exercise ergometer (see Figure 14), use of which will be included in the 1-day and 3-day "Day-in-the-Life" timelines. The device and its integration within the LER will be included in the human factors evaluation of the LER crew accommodations. Crewmembers will wear heart rate monitors during exercise and will not exceed $85 \%$ of age-predicted maximum heart rate. NASA GRC provided the ergometer. [4]

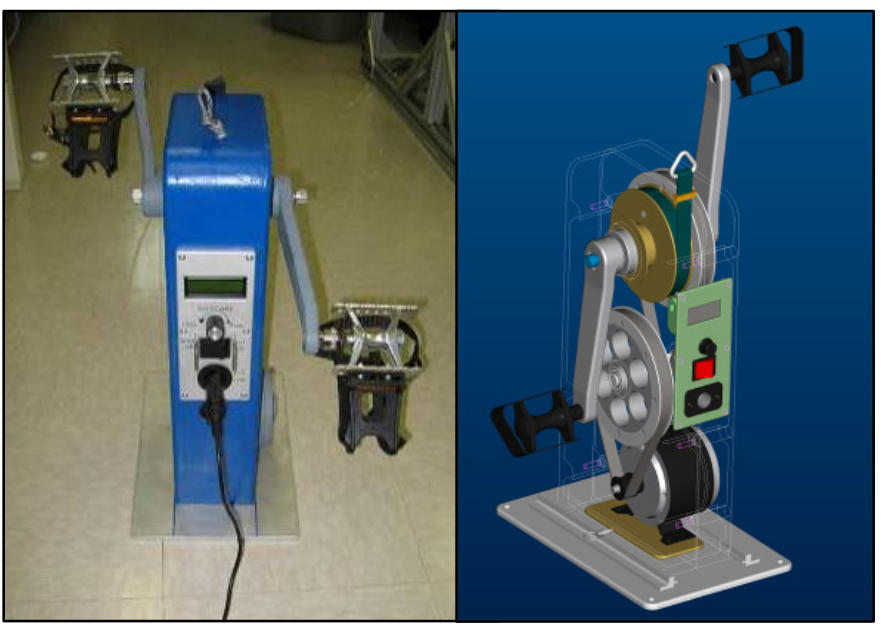

Figure 14: LER exercise ergometer
COMMUNICATIONS, NETWORKING, AND NAVIGATION INFRASTRUCTURE

NASA KSC's Information Technology and Communications Directorate was responsible for engineering, implementing, testing, and deploying operationally a diverse communications and networking infrastructure to support test operations. This infrastructure is known as a Lunar Communications and Network Emulator (LCNE), and the system was deployed to model an Agency Lunar Comm and Network architecture to evaluate the architecture and identify gaps in planning. The architecture that was modeled at D-RATS 2008 is illustrated in figure 15. Space suit, LER, mission control teams (e.g. CAPCOM), science operations personnel (e.g. Science CAPCOM), as well as other researchers, utilized the system deployed by this team to conduct their field testing.

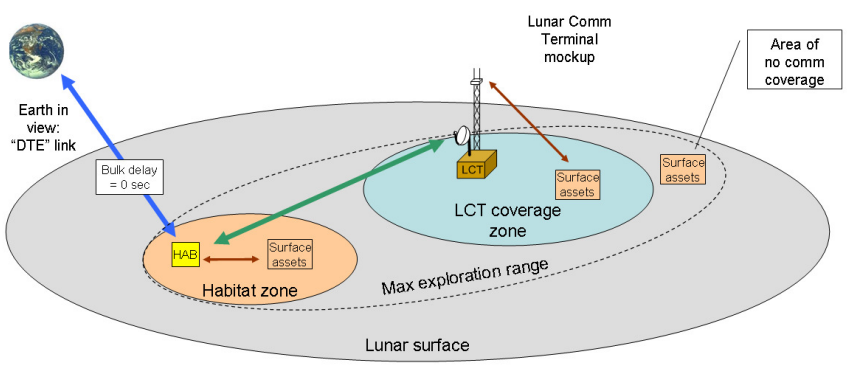

Figure 15: Lunar comm architecture modeled at $D$ RATS 2008 analog test

The team provided the following major areas of support:

- Installed comm and networking assets on a nearby Elden mountain to serve as aggregate broadband network bandwidth to the field test from a commercial Internet Service Provider (ISP)

- Secured the network from Internet attacks, and tunneled test site traffic back to NASA's networks

- Measured network utilization and performance useful for future lunar data requirements planning

- Engineered and deployed a duplicate network tunnel from a JSC location to allow engineers to test their field configuration prior to the outing during dry run operations

- Engineered, tested, and deployed the entire test site video, voice, and data network, which included multiple routers, switches, and even tactical fiber deployment at the test site

- Engineered, fabricated, tested and deployed a mockup Lunar Communication Terminal (LCT)

- Provided Spectrum Management leadership for the entire D-RATS test team

- Engineered, fabricated, tested, and deployed pan/tilt/zoom cameras that were remotely controlled throughout the field test

- Engineered and fielded on-suit EVA megapixel science cameras and associated science CAPCOM image and video stream data display system 
- Assisted with design and testing of LER rover audio and radio system

- Validated performance of a low data rate $\mathrm{Hi}$ Definition mobile camera standard for lunar rovers and suits

Navigation and asset tracking and recording for the rovers at the field test was facilitated through the use of the United States (US) GPS.

\section{TEST OBJECTIVES}

Each year, specific task activities are selected to support the overarching goal of exploration EVA system requirements development. The "Test Results and Discussion" section of this paper describes the test results for each objective.

The focus of Desert RATS 2008 was to establish and capture baseline engineering operational performance characteristic metrics between the UPR and LER vehicle configurations while under extended periods of representative mission-based scenario test operations. This included evaluation of the productivity of the LER compared to a UPR on 1-day geological/mapping traverses, evaluation of the human factors and crew accommodations of the LER and suit ports on 1-day and 3-day geological traverses, evaluation of a single person EVA capability, and assessing night-time operations of the LER and UPR.

Primary objectives:

The primary test objectives were to:

1) Gather data for 1-day "day in the life" surface mission sorties using the LER and UPR

2) Gather data for an extended 3-day (including overnight stays) surface mission sortie using the LER

These two objectives encompass the following engineering objectives:

- Perform night-time driving and exploration / mapping / geological sample collection and documentation tasks using LER and UPR vehicles.

- Measure performance metrics during 1-day and 3day (LER only) exploration / mapping / geological traverses, which will be compared with assumptions in the CXAT_Lunar power-mobility models and Human Research Program (HRP) models of physiological adaptations during long-term lunar sorties.

- Develop, revise and refine procedures, processes, mission rules, traverse planning techniques and data collection and analysis methods for UPR, LER, and planetary EVA operations. [4]
Secondary objectives:

The secondary test objectives were to:

3) Evaluate operational impacts of lunar surface communications scenarios involving the use of a simulated lunar comm terminal, a direct to earth link, and the function of a lunar relay satellite

4) Provide a PAO opportunity for students and media

The primary objectives of this study have been decomposed into specific hypotheses which were tested with a combination of objective and subjective productivity, performance, and human factors metrics. These hypotheses are detailed below. The study design incorporates a direct cross over comparison between the LER and the UPR, using two different crews, each made up of a professional field geologist and an active NASA astronaut with EVA experience. [4]

Hypotheses:

1) Productivity/Performance achieved during 1day exploration / mapping / geological traverses using the LER will be equal to or greater the productivity/performance achieved during UPR traverses, with less suit time.

2) Range achieved during 1-day exploration / mapping / geological traverses in the LER will be greater than during 1-day UPR traverses.

3) Subjective assessment of contextual observations from inside an LER will be equal to unsuited contextual observations.

4) Human interfaces to the LER suit ports and alignment guides will be acceptable as assessed by human factors metrics.

5) The human factors and crew accommodations within the LER will be acceptable to support a 3-day exploration / mapping / geological traverse.

6) Single-person EVA from the LER exploiting the advantages of IVA and EVA crewmembers will result in productivity equal to or greater than a two-person EVA from the LER or UPR, with less EVA suit time.

\section{TEST SCHEDULE}

Preparing for a Desert RATS remote field test takes a year of planning, preparation, hardware development, and dry run tests. 
The remote field test was the last two weeks of October 2008. The first week focused on the 1-day "day in the life" scenarios with the LER and UPR, Media Day, and educational outreach at the test site. The second week focused on the extended 3-day LER mission.

\section{TEST RESULTS AND DISCUSSION}

OBJECTIVES \#1 AND 2:

1) Gather data for 1-day "day in the life" surface mission sorties using the LER and UPR

2) Gather data for an extended 3-day (including overnight stays) surface mission sortie using the LER

\section{$\underline{\text { Test Results: }}$}

1-day UPR traverses had an 8 hour duration. 1-day LER traverses had a 15 hour duration. Each day of the 3-day LER traverse lasted 15 hours.

There are several limitations to the data that is presented:

- Testing is not designed to evaluate pressure suit human factors

- Flight-like suit port egress/ingress procedures and mechanisms were used, but suits and suit ports were not pressurized

- Testing was performed in a 1-g environment

- Practical constraints precluded balancing the order of rover configurations to eliminate possible learning biases

Hypothesis \#1: Productivity/Performance achieved during 1-day exploration / mapping / geological traverses using the LER will be equal to or greater the productivity/performance achieved during UPR traverses, with less suit time.

Productivity/Performance and EVA suit time data were collected during two 1-day UPR traverses and two 1-day LER traverses.

Productivity/Performance was measured as the sum of (Value of Traverse Objective x Data Quality) for all traverse objectives.

Results show that the average time spent EVA in the LER was $61 \%$ less than in the UPR and that the average productivity/performance in the LER was $57 \%$ greater than in the UPR (figure 16). The LER productivity/performance per EVA hour was an average of 4.7 times more productive per EVA hour than the UPR (figure 17). Therefore, the hypothesis was accepted.
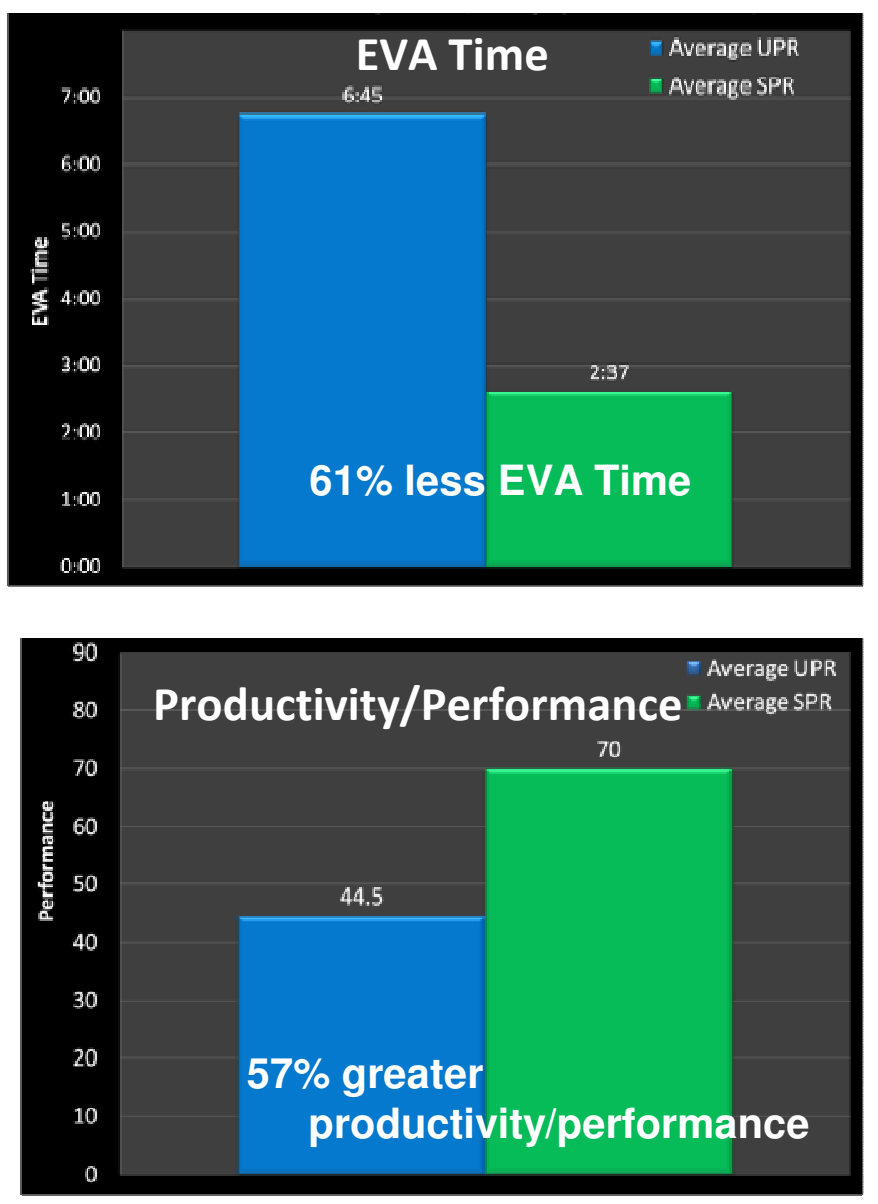

Figure 16: EVA time and productivity/performance results for hypothesis \#1

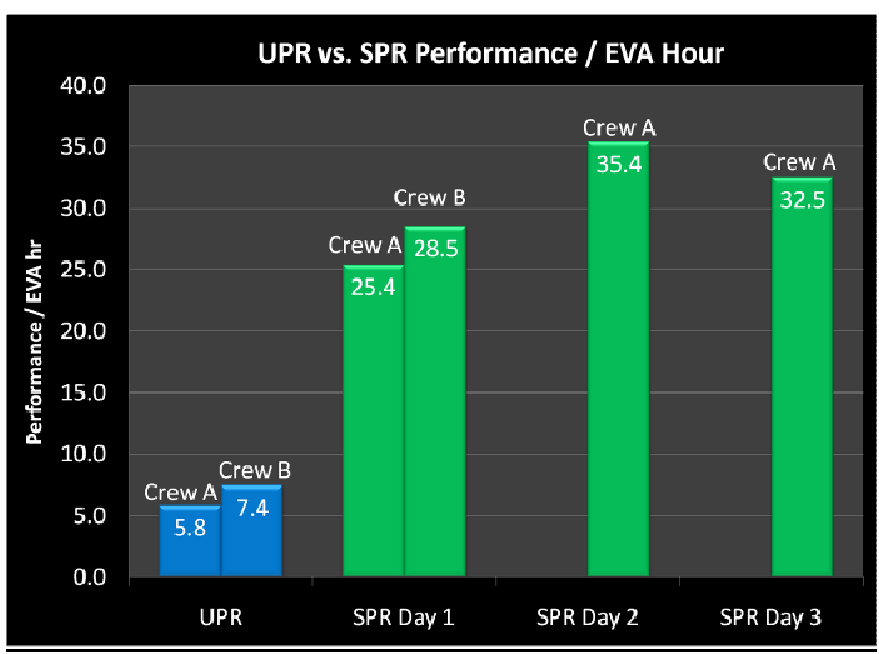

Figure 17: UPR vs. LER productivity/performance per EVA hour results for hypothesis \#1

Hypothesis \#2: Range achieved during 1-day exploration / mapping / geological traverses in the LER will be greater than during 1-day UPR traverses.

Range data (GPS-derived) was collected during two 1day UPR traverses and two 1-day LER traverses. 
Results show that the average LER traverse distance was $18.2 \mathrm{~km}$ and the average UPR traverse distance was $13.9 \mathrm{~km}$. The traverse ranges for both LER and UPR were about the same at $5.3 \mathrm{~km}$ and $5.0 \mathrm{~km}$, respectively (figure 18). Due to communication coverage limitations at this year's test, the maximum range of traverses was restricted. The hypothesis was accepted.

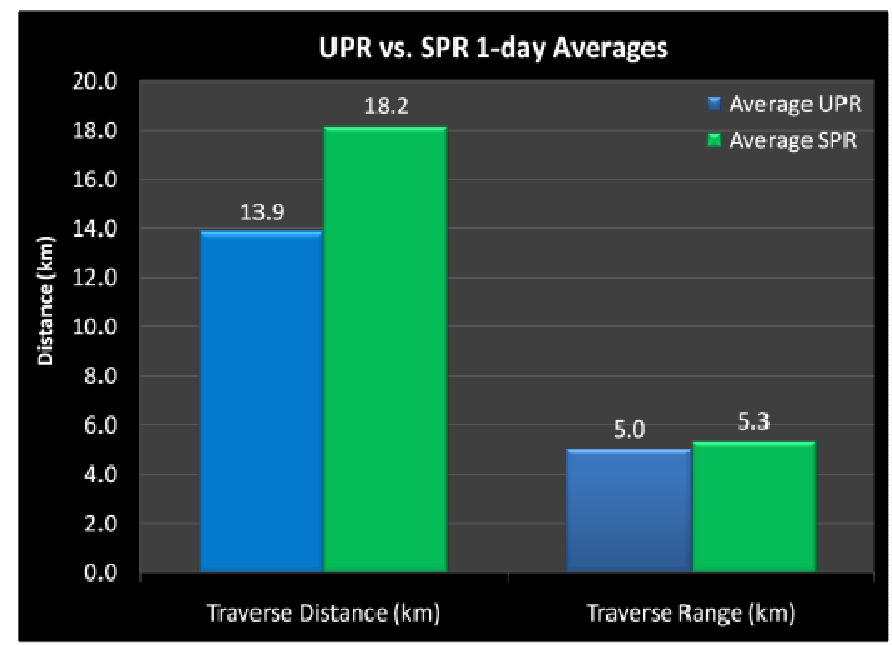

Figure 18: Traverse distance and range UPR vs. LER 1day average results for hypothesis \#2

Hypothesis \#3: Subjective assessment of contextual observations from inside an LER will be equal to unsuited contextual observations.

Four geologists were allowed up to 20 minutes to make shirt-sleeved observations of an area of the BPLF. They were then allowed up to 20 minutes to make observations from within the LER, then they rated the geological observation quality from inside the LER and provided other subjective remarks.

Results show that the average geological observation quality inside the LER was 2.9 and the shirt-sleeve geological observation quality was 3.0. This suggests that for this terrain, the quality of contextual observations from inside the LER is approximately equal to unsuited contextual observations. The hypothesis was accepted. Figure 19 shows a geologist making observations from within the LER.

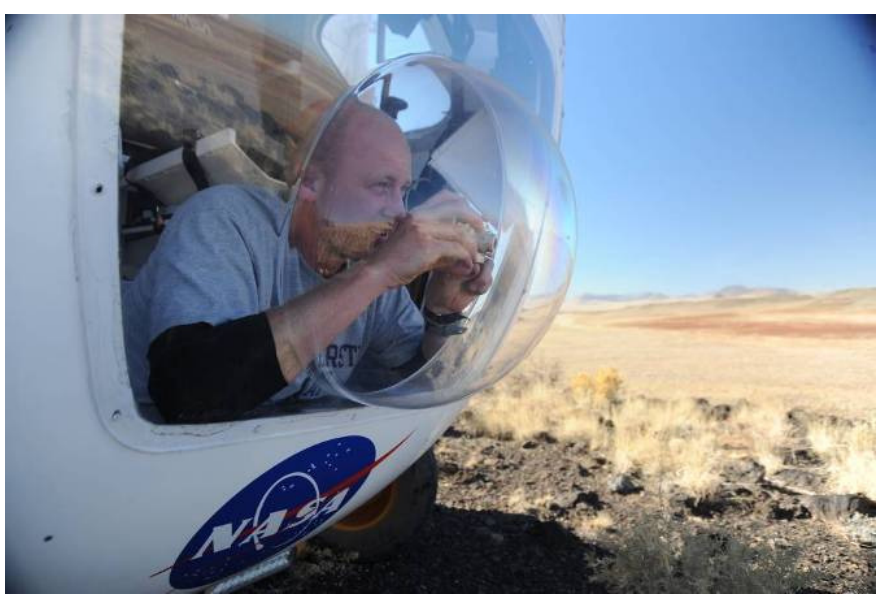

Figure 19: Geologist making observations from inside the $L E R$

Hypothesis \#4: Human interfaces to the LER suit ports and alignment guides will be acceptable as assessed by human factors metrics.

Suit port human factors data was collected from four subjects during 5 days of LER traverses.

The data is currently being analyzed. Preliminary analysis, including data collected during dry runs at JSC, suggests that the human factors of the suit ports and alignment guides are acceptable (figure 20). Redesign of the suit port mechanism controls and external alignment guides to be more easily operated could further increase performance and productivity. There were no issues with the internal cabin volume for suit donning and doffing, but minor improvements were suggested for internal access to the suit port and general operations. Suit port egress times averaged 11 minutes \pm 3 minutes and ingress times averaged 5 minutes \pm 1 minute. The test subjects showed that the suit port concept was practical, viable, and a highly efficient way to perform EVA operations from the LER, so the hypothesis was accepted.

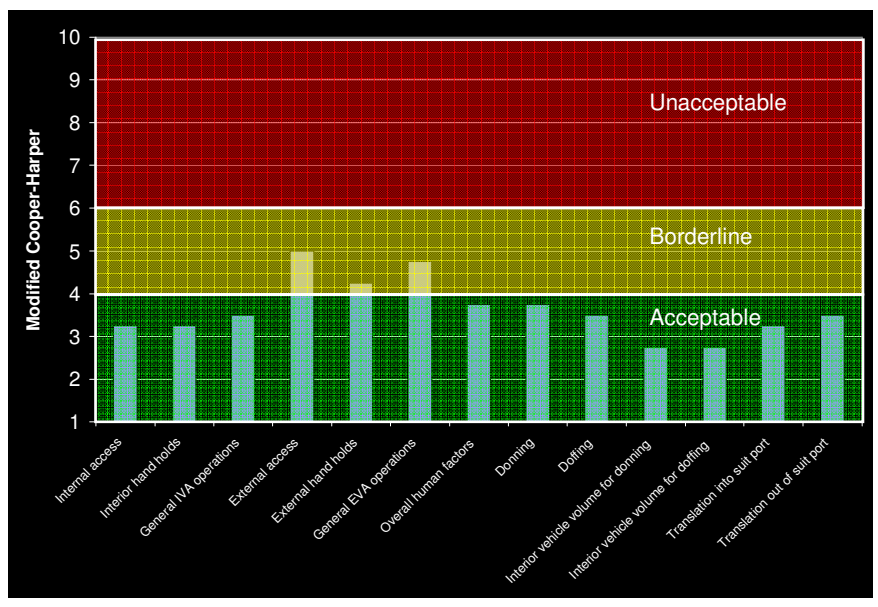

Figure 20: Suit port human factors data for hypothesis \#4 
Hypothesis \#5: The human factors and crew accommodations within the LER will be acceptable to support a 3-day exploration / mapping / geological traverse.

Pre and post fatigue data and displays and controls ratings were collected for two 1-day UPR traverses, two 1-day LER traverses, and each day of the 3-day LER traverse. Data was collected using a 1 to 10 scale (figure 21).

\begin{tabular}{|c|c|c|c|c|c|c|c|c|c|}
\hline \multicolumn{2}{|c|}{$\begin{array}{l}\text { No Fatigue: } \\
\text { performance not } \\
\text { compromised }\end{array}$} & \multicolumn{2}{|c|}{$\begin{array}{l}\text { Minor Fatigue: } \\
\text { performance not } \\
\text { compromised }\end{array}$} & \multicolumn{2}{|c|}{\begin{tabular}{|c|} 
Moderate Fatigue: \\
performance will likely \\
be compromised if \\
continued
\end{tabular}} & \multicolumn{2}{|c|}{$\begin{array}{l}\text { Significant Fatigue: } \\
\text { performance is } \\
\text { compromised }\end{array}$} & \multicolumn{2}{|c|}{$\begin{array}{c}\text { Extreme Fatigue: } \\
\text { unable to continue } \\
\text { with adequate } \\
\text { performance }\end{array}$} \\
\hline 1 & 2 & 3 & 4 & 5 & 6 & 7 & 8 & 9 & 10 \\
\hline
\end{tabular}

Figure 21: Scale used for hypothesis \#5

Results show that the fatigue level after a 1-day UPR traverse was greater than after a 1-day LER traverse (moderate vs. minor, see figure 22). Fatigue during the 3-day LER traverse was between no fatigue and minor fatigue (figure 23). Overall, the displays and controls were rated borderline, suggesting that improvements are warranted. The hypothesis was accepted.

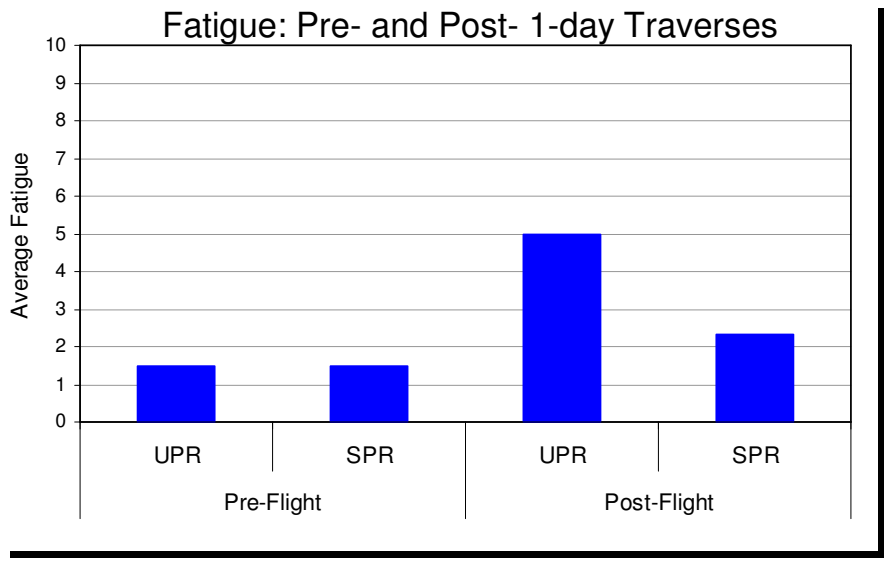

Figure 22: Pre and post fatigue ratings for 1-day UPR and LER traverses

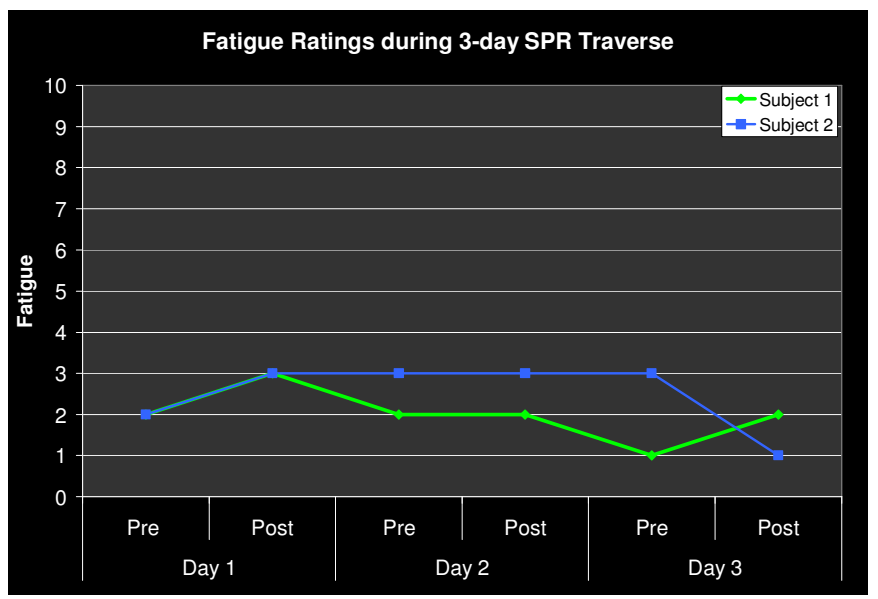

Hypothesis \#6: Single-person EVA from the LER exploiting the advantages of IVA and EVA crewmembers will result in productivity equal to or greater than a twoperson EVA from the LER or UPR, with less EVA suit time.

No dedicated protocol was performed to compare 1person and 2-person EVAs. However, data collected during UPR and LER traverses allows comparison of performance at the same sites with 1-person and 2person EVAs. The data is currently being analyzed. Figure 24 shows a 1-person EVA from the LER at night.

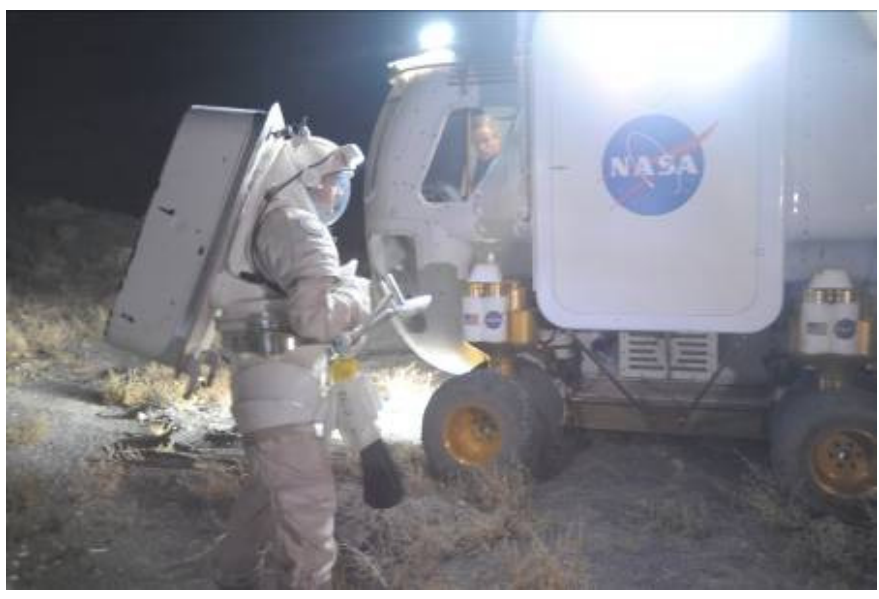

Figure 24: 1-person EVA from the LER at night

\section{OBJECTIVE \#3:}

Evaluate operational impacts of lunar surface communications scenarios involving the use of a simulated lunar comm terminal, a direct to earth link, and the function of a lunar relay satellite.

\section{Test Results:}

The data communications architecture employed were nominal for $10 \mathrm{~km}$ traverses from the outpost. The voice communications architecture was also nominal for $10 \mathrm{~km}$ traverses from the outpost, but full-duplex voice communications are required for future tests between the LER and suits and the Earth-based mission control. Two voice loops were employed for the test (Flight and Engineering loops), and more loops are required to split sub-teams into private coordination loops.

Use of the US GPS system was adequate for the purposes of navigating traverses during this test, and recording the progress operation. Future tests should involve the employment of navigation techniques that more accurately model the lunar navigation architecture.

OBJECTIVE \#4:

Provide a PAO opportunity for students and media

Figure 23: Fatigue ratings during 3-day LER traverse 
Wednesday, October 22 had a designated time slot for an educational outreach event where $\sim 100$ students toured the hardware at the BPLF test site and met the two astronaut test subjects. Figure 25 shows some of the students as they listened to astronauts Mike Gernhardt and Rex Walheim.

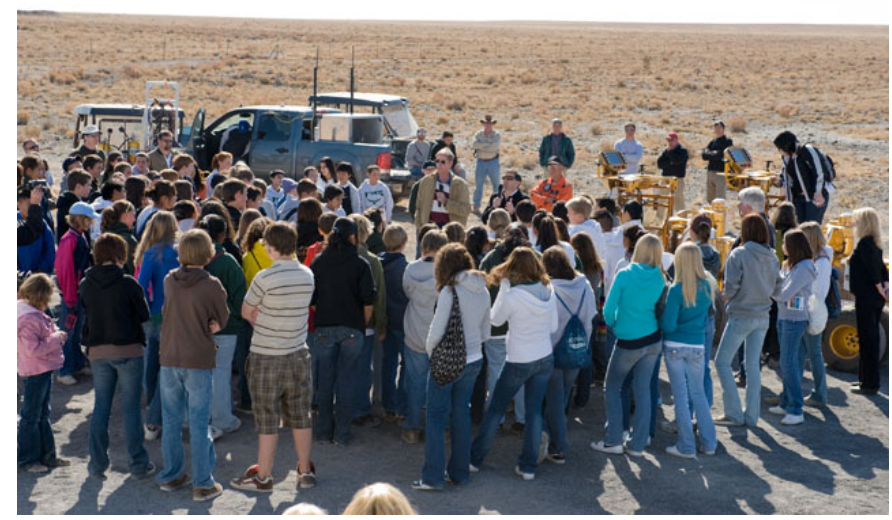

Figure 25 (jsc2008e138677): Students during educational outreach event

Friday, October 24 was designated as "Media Day," a day that television and print reporters could film parts of the tests and interview lead engineers from the various projects represented. There was good representation from the media outlets listed below. NASA EDGE also participated.

- Fox News

- Reuters

- Arizona Daily Sun

- Arizona Republic

- KNXV ABC15

- China News Service

- China Central TV

- Russian State TV

- Ideacom

\section{CONCLUSION}

Exploration system requirements development, as manifested in the RATS and its desert field test, is an important on-going process. As pressure to focus on near-term flight hardware goals increases, a concerted effort to continue investments in planetary exploration requirements development, technology research and incubation, and personnel experience and skills development should be maintained. A coordinated, NASA-wide approach to analog testing would sustain the existing momentum and focus the products of field testing to the benefit of the Constellation program.
The authors would like to acknowledge all of the D-RATS 2008 participants, especially those who supported this paper by authoring and/or reviewing their respective sections:

- Jesse Buffington/JSC

- Marc Seibert/KSC

Thank you for your enthusiasm and dedication. As always, it is a pleasure to work with you!

Thanks go to Regan Geeseman, our outstanding NASA photographer, to whom belongs the credit for the "jsc2008" numbered photographs.

\section{REFERENCES}

1. Romig, Barbara, Kosmo, J., Ross, A., Bernard, C., Aitchison, L., Eppler, D., and Splawn, K. "Desert Research and Technology Studies 2006 Report." $37^{\text {th }}$ International Conference on Environmental Systems. 2007-01-3131. July, 2007. Chicago, IL.

2. Kosmo, Joseph, Janoiko, B., and Eppler, D. "Desert RATS (Research and Technology Studies) Analog Test Areas." Space Technology and Applications International Forum (STAIF). February, 2007. Albuquerque, NM.

3. http://www.nasa.gov/centers/johnson/news/releases/ 2008/H08-068.html

4. Gernhardt, Michael L., Abercromby, A., Ambrose, R., Howard, R., Kosmo, J., Ney, Z., and Romig, B. "Engineering Evaluation of Small Pressurized Rover and Unpressurized Rover Configurations during Simulated Planetary Surface Exploration." August 28, 2008.

5. Romig, Barbara and Allton, C. "First Generation Suit Port Development and Testing." Johnson Space Center Engineering Directorate Biennial Report. February, 2009.

6. Romig, Barbara and Kosmo, J. "Desert Research and Technology Studies 2007 Report." $38^{\text {th }}$ International Conference on Environmental Systems. 2008-01-2062. July, 2008. San Francisco, CA.

\section{CONTACT}

For further information, past field test reports, or to express interest in future potential Desert RATS participation, please contact:

Joseph J. Kosmo

NASA Johnson Space Center

joseph.j.kosmo@nasa.gov

Barbara A. Romig

NASA Johnson Space Center

barbara.a.romig@nasa.gov 


\section{ACRONYMS}

- AEVA Advanced Extra Vehicular Activity

- ARC Ames Research Center

- ASU Arizona State University

- BPLF Black Point Lava Flow

- CXAT_Lunar

Constellation Lunar Architecture Team

- D-RATS Desert Research and Technology Studies

- $E A$

- EC

- ER

- EVA

- GPS

- $\mathrm{GRC}$

- $\mathrm{HQ}$

- HRP

- HUT
Human Research Program

Hard Upper Torso
- ISP

- IVA

- JSC

- KSC

- LaRC

- LCNE

- LCT

- LER

- LPI

- NASA

- PAO

- PLSS

- RATS

- UPR

- US

- USGS

- UTAF
Internet Service Provider

Intravehicular Activity

Johnson Space Center

Kennedy Space Center

Langley Research Center

Lunar Communications and Network Emulator

Lunar Communication Terminal

Lunar Electric Rover

Lunar Planetary Institute

National Aeronautics and Space

Administration

Public Affairs Office

Portable Life Support System

Research and Technology Studies

Unpressurized Rover

United States

United States Geologic Survey

Usability, Testing, and Analysis Facility 